OPEN ACCESS

Edited by:

Hongji Dai,

Tianjin Medical University Cancer Institute and Hospital, China

Reviewed by: Zanxian Xia, Central South University, China Jia-Yuh Chen, Chung Shan Medical University,

Taiwan

*Correspondence: Dayan Wang dayanwang@cnic.org.cn Yuelong Shu, shuylong@mail.sysu.edu.cn

Specialty section: This article was submitted to Applied Genetic Epidemiology,

a section of the journal

Frontiers in Genetics

Received: 15 June 2021 Accepted: 27 September 2021

Published: 22 October 2021

Citation:

LiM, Wei H, Zhong S, Cheng Y, Wen S, Wang D and Shu Y (2021) Association of Single Nucleotide Polymorphisms in LEP, LEPR, and PPARG With Humoral Immune Response to Influenza Vaccine.

Front. Genet. 12:725538. doi: 10.3389/fgene.2021.725538

\section{Association of Single Nucleotide Polymorphisms in LEP, LEPR, and PPARG With Humoral Immune Response to Influenza Vaccine}

\author{
Mao $\mathrm{Li}^{1}$, Hejiang Wei ${ }^{2}$, Shuyi Zhong ${ }^{1}$, Yanhui Cheng ${ }^{2}$, Simin Wen ${ }^{1}$, Dayan Wang ${ }^{2 \star}$ and \\ Yuelong Shu ${ }^{1 *}$
}

${ }^{1}$ School of Public Health (Shenzhen), Shenzhen Campus of Sun Yat-sen University, Guangzhou, China, ${ }^{2}$ National Institute for Viral Disease Control and Prevention, Chinese Center for Disease Prevention and Control, Beijing, China

Background: Although previous studies have proposed leptin plays an important role in energy metabolism as well as in immune response, the effects of leptin-related genes on influenza vaccine-induced immune response remain unexplored. In this study, we aimed to investigate the potential association of leptin gene ( $L E P)$, leptin receptor gene $(L E P R)$, and peroxisome proliferator activated receptor gamma gene (PPARG) polymorphisms with humoral immune response to influenza vaccine.

Methods: Based on the seroconversion to influenza vaccine, 227 low-responders and 365 responders were selected in this study, and 11 candidate single nucleotide polymorphisms (SNPs) were genotyped using the MassARRAY technology platform. Univariate and multivariate logistic regression analyses were used to explore the association of SNPs in LEP, LEPR, and PPARG with humoral immune response to influenza vaccine. We also conducted a stratified analysis by gender to further clarify this association. The haplotypes analysis was performed using SNPStats.

Results: Significant differences were observed in the genotypic distribution of PPARG rs17793951 between the two groups ( $p=0.001)$, and the PPARG rs17793951 AG + GG genotype was associated with a higher risk of low responsiveness to influenza vaccine adjusted for gender and age (additive genetic model: $\mathrm{OR}=2.94,95 \% \mathrm{Cl}=1.67-5.19$, dominant genetic model: $\mathrm{OR}=2.81,95 \% \mathrm{Cl}=1.61-4.92)$. No significant association of other SNPs in $L E P$ and $\angle E P R$ with immune response to influenza vaccine was found. The stratified analysis found the gender difference in the association of LEPR and PPARG variants with immune response to influenza vaccine. We found that $L E P R$ rs6673591 GA + AA genotype was correlated with low responsiveness to influenza vaccine only in males $(\mathrm{OR}=1.96,95 \% \mathrm{Cl}=1.05-3.67)$, and PPARG rs17793951 AG + GG genotype was associated with low responsiveness to influenza vaccine in females $(\mathrm{OR}=3.28,95 \% \mathrm{Cl}=$ 1.61-6.67). Compared with the CGGAGGC haplotype composed of LEPR rs1327118, rs7602, rs1137101, rs 1938489, rs6673591, rs1137100, and rs13306523, the CAAAAAC haplotype was positively correlated with immune response of influenza vaccine (OR $=0.34$, $95 \% \mathrm{Cl}=0.15-0.77$ ). Haplotype TG comprised of PPARG rs796313 and rs17793951 was associated with a 2.85 -fold increased risk of low responsiveness to influenza vaccine. 
Conclusion: Our study identified that PPARG rs17793951 variants were significantly associated with the immune response to influenza vaccine.

Keywords: influenza vaccine, single nucleotide polymorphism (SNP), LEP, LEPR, PPARG, immune response

\section{INTRODUCTION}

Influenza is an infectious respiratory disease transmitted by droplets or human-to-human close contacts. It is estimated that the seasonal influenza epidemic causes one billion cases worldwide every year, including three to five million severe cases and 290,000-650,000 deaths (Iuliano et al., 2018). Influenza vaccination is the most effective measure to prevent influenza infection, which is recommended for pregnant women, children, the elderly, health care workers, and individuals with specific chronic diseases in priority. The most commonly used influenza vaccine is trivalent inactivated influenza virus split vaccine, containing two influenza $\mathrm{A}$ antigens $(\mathrm{A} / \mathrm{H} 1 \mathrm{~N} 1$ and $\mathrm{A} / \mathrm{H} 3 \mathrm{~N} 2)$ and one influenza $\mathrm{B}$ antigen $(\mathrm{B} /$ Victoria or $\mathrm{B} /$ Yamagata). Some studies have suggested that $0.3 \%-10 \%$ of healthy individuals with influenza vaccination still failed to elicit protective antibodies (Narang et al., 2018; Soedjatmiko et al., 2018). Several host factors play important roles in the regulation of humoral response to influenza vaccine, such as age, gender, health status, pre-existing immunity, and genetic factors (Dhakal and Klein, 2019). Among the genetic factors, many single nucleotide polymorphisms (SNPs) in human leucocyte antigen (HLA), cytokine, and cytokine receptor-related genes have been confirmed to be significantly associated with humoral response to influenza vaccine (Poland et al., 2008; Franco et al., 2013).

Leptin (LEP), an adipocyte-derived hormone, is known for its ability to regulate energy metabolism and immune response (Francisco et al., 2018). The immune-modulating effect of leptin starts with its binding to the leptin receptor (LEPR) expressed on the membrane of immune cells. It is reported that leptin regulates the proliferation and reactivity of $\mathrm{T}$ cells by activating the JAK/STAT pathway to influence cellular immunity (Kim et al., 2010). Moreover, leptin participates in the humoral immune response by up-regulation the expressing of TNF- $\alpha$, IL-6, and IL-10 (Agrawal et al., 2011). In addition, peroxisome proliferator activated receptor gamma (PPARG) coordinates with leptin and also plays a role in adipocyte differentiation and inflammatory response in protein interaction network (Joffe and Houghton, 2016). There are many SNPs in the promoter, intron, and exon regions of $L E P, L E P R$, and $P P A R G$, which might regulate related genes transcription, splicing, and translation. Previous studies have reported that some leptin-related gene polymorphisms were associated with the influenza antibody levels to influenza vaccine in elderly Caucasians (Ovsyannikova et al., 2014). Nevertheless, the association between SNPs in LEP, LEPR, and PPARG and influenza vaccine-induced immune response has not been revealed in the Chinese Han population.

In this study, we detected 11 tag SNPs in 592 subjects and hypothesized that certain SNPs may explain the poor immunogenicity of influenza vaccine. This study aimed to elucidate the association of SNPs in LEP, LEPR, and PPARG with humoral immune response to influenza vaccine in the Chinese Han population.

\section{MATERIALS AND METHODS}

\section{Study Design and Subjects}

In total, 1968 healthy volunteers were consecutively recruited in this study at Xinjiang Uygur Autonomous Region and Yunnan Province, China, from September 2009 to September 2019. All subjects were only vaccinated one intramuscular dose of trivalent inactivated seasonal influenza vaccine (TIV, Fluarix, $0.50 \mathrm{ml}$ ), which was manufactured according to the World Health Organization (WHO) recommendation for the northern hemisphere. Subsequently, 386 subjects were excluded for non-Han Chinese, loss to follow-up, inadequate blood samples, and repeat vaccinations. The flow diagram of this study was shown in Figure 1. We finally selected 592 individuals according to the seroconversion to $\mathrm{A} / \mathrm{H} 1 \mathrm{~N} 1, \mathrm{~A} / \mathrm{H} 3 \mathrm{~N} 2$, and $\mathrm{B}$ vaccine components, including 227 low-responders (achieved seroconversion to none of vaccine components) and 365 responders (achieved seroconversion to all vaccine components). A case-control study design was conducted to analyze the association of SNPs in LEP, LEPR, and PPARG with humoral response to influenza vaccine in the Chinese Han population. Questionnaires were collected to obtain personal information, such as gender, age, vaccine type, vaccination history, etc. Peripheral venous blood samples were collected prior to (day 0) and after (day 28) vaccination and stored at $-30^{\circ} \mathrm{C}$.

\section{SNP Selection and Genotyping}

Eleven tag SNPs in $L E P, L E P R$, and PPARG were selected using dbSNP database (https://www.ncbi.nlm.nih.gov/snp/) and SNPinfo Web Server (https://manticore.niehs.nih.gov/) with minor allele frequency $(\mathrm{MAF})>0.05$ in the Chinese Han Beijing (CHB) population and $r^{2}>0.8$, including rs2167270 and rs11761556 in $L E P$, rs1327118, rs7602, rs1137101, rs1938489, rs6673591, rs1137100, and rs13306523 in $L E P R$, and rs796313 and rs17793951 in PPARG (Supplementary Table 1). Genome DNA was extracted using an AxyPrep Blood Genomic DNA Miniprep kit (Axygen, Union City, California, United States) according to the manufacturer's instructions. Genotyping of candidate SNPs was conducted using the MassARRAY technology platform (Sequenom, San Diego, California, United States) and implemented by BioMiao Biological Technology (Beijing, China). As shown in Supplementary Table 2, the call rates for 11 tag SNPs in $L E P, L E P R$, and PPARG were all higher than $98.0 \%$. Additionally, we used HaploReg (V.4.1, https:/pubs.broadinstitute.org/mammals/ haploreg/haploreg.php) to explore protein binding annotations of SNPs.

\section{Influenza Hemagglutination Inhibition (HAl) Assay}

The HAI assays were used to measure sera antibody titers with $1 \%$ solution of turkey red blood cells against influenza vaccine strains according to standard methods described in 


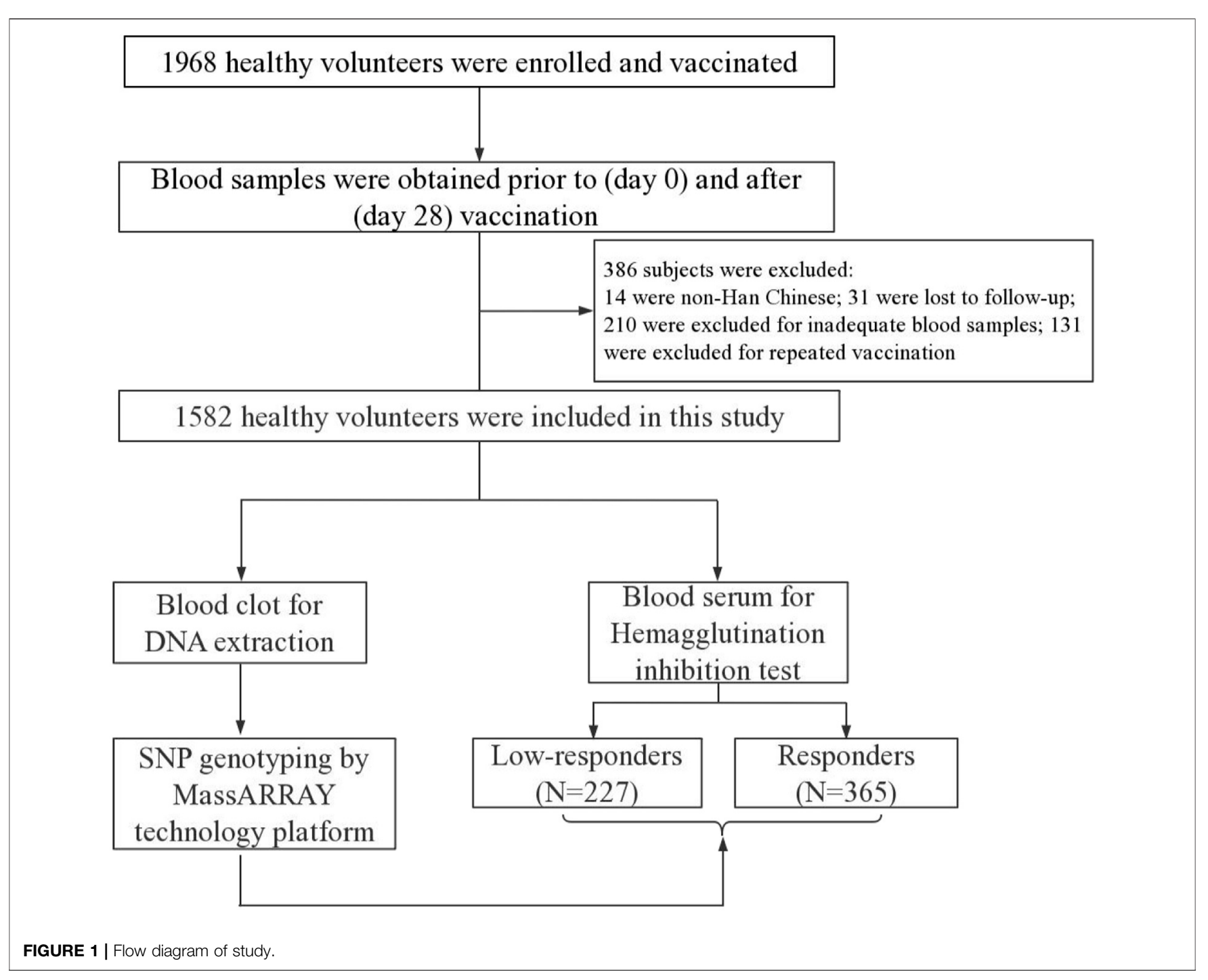

the WHO guideline (World Health Organization, 2011World Health Organization, 2011). The vaccine strains recommended by WHO for the northern hemisphere from 2009-2010 to 2019-2020 flu seasons are summarized in Supplementary Table 3. Seroconversion was defined as either post-vaccination HAI titer $\geq 40$ if pre-vaccination HAI titer $<10$ or at least fourfold increase if prevaccination $\mathrm{HAI}$ titer $\geq 10$. Moreover, seroconversion factors (SCFs) were considered as the fold rises of geometric mean titer (GMT) of HAI antibody titers postvaccination.

\section{Statistical Analysis}

The Hardy-Weinberg equilibrium (HWE) test was conducted to assess the genotype frequencies of SNPs among subjects. Continuous variables with normal distribution through the Kolmogorov-Smirnov test were described as the $\overline{\mathrm{x}} \pm \mathrm{s}$ and analyzed by One-way ANOVA (analysis of variance).
Categorical data was summarized as frequencies (percentages) and compared using $\chi^{2}$ test or Fisher's exact test appropriately. Associations of tag SNPs with immune response to influenza vaccine were analyzed using univariate and multivariate logistic regression models adjusted for gender and age in additive genetic model and dominant genetic model, and the odds ratios (95\% CIs) were also estimated. We also conducted a stratified analysis to explore this association in females or males, separately. The pairwise linkage disequilibrium (LD) and haplotype analysis were performed using SNPStats (http://bioinfo.iconcologia.net/ SNPStats). Gene-gene interactions were calculated with the GMDR program (V.0.7, http://sourceforge.net/projects/gmdr/). All analyses were performed by the SPSS software (V.25.0) and GraphPad Prism software (V.6.01), and the significance level was set at $p<0.05$ with two-tailed. When we compared the genotypic frequencies of 11 SNPs between the two groups, the significance level was set at $p<0.0045(0.05 / 11)$ after the Bonferroni corrections. 
TABLE 1 | The genotypic frequencies and odds ratios of SNPs in $L E P, L E P R$, and PPARG between the two groups in additive genetic model and dominant genetic model.

\begin{tabular}{|c|c|c|c|c|c|c|c|}
\hline Gene & SNP ID & Genotype & Low-responders $\mathrm{N}^{\mathrm{a}}(\%)$ & Responders $\mathbf{N}^{\mathrm{a}}(\%)$ & OR $(95 \% \text { Cl })^{b}$ & OR $(95 \% \mathrm{Cl})^{\mathrm{c}}$ & $P_{H W E}$ \\
\hline \multirow[t]{8}{*}{ LEP } & rs2167270 & $\mathrm{GG}$ & $159(71.0)$ & $232(64.3)$ & 1.00 (reference) & 1.00 (reference) & 0.737 \\
\hline & & $A G$ & $58(25.9)$ & $118(32.7)$ & $0.72(0.49-1.04)$ & $0.72(0.50-1.05)$ & \\
\hline & & $\mathrm{AA}$ & 7 (3.1) & $11(3.0)$ & $0.93(0.35-2.45)$ & $1.10(0.41-2.96)$ & \\
\hline & & $A G+A A$ & $65(29.0)$ & $129(35.7)$ & $0.74(0.51-1.05)$ & $0.75(0.52-1.08)$ & \\
\hline & rs11761556 & $\mathrm{AA}$ & $129(57.1)$ & $197(54.6)$ & 1.00 (reference) & 1.00 (reference) & 0.612 \\
\hline & & $A C$ & 88 (38.9) & 138 (38.2) & 0.97 (0.69-1.38) & 0.98 (0.69-1.39) & \\
\hline & & $\mathrm{CC}$ & $9(4.0)$ & $26(7.2)$ & $0.53(0.24-1.17)$ & $0.58(0.26-1.28)$ & \\
\hline & & $A C+C C$ & $97(42.9)$ & $164(45.4)$ & $0.90(0.65-1.26)$ & $0.92(0.65-1.28)$ & \\
\hline \multirow[t]{28}{*}{ LEPR } & rs1327118 & $\mathrm{CC}$ & $160(70.8)$ & $270(74.8)$ & 1.00 (reference) & 1.00 (reference) & 0.956 \\
\hline & & CG & $63(27.9)$ & $82(22.7)$ & 1.30 (0.89-1.90) & 1.35 (0.92-1.98) & \\
\hline & & GG & 3 (1.3) & $9(2.5)$ & $0.56(0.15-2.11)$ & $0.58(0.15-2.20)$ & \\
\hline & & $C G+G G$ & $66(29.2)$ & $91(25.2)$ & $1.22(0.84-1.78)$ & $1.27(0.87-1.85)$ & \\
\hline & rs7602 & GG & $165(73.0)$ & $255(70.2)$ & 1.00 (reference) & 1.00 (reference) & 0.946 \\
\hline & & GA & $56(24.8)$ & $99(27.3)$ & $0.87(0.60-1.28)$ & $0.89(0.61-1.31)$ & \\
\hline & & $\mathrm{AA}$ & $5(2.2)$ & $9(2.5)$ & $0.86(0.28-2.61)$ & $0.86(0.28-2.64)$ & \\
\hline & & $\mathrm{GA}+\mathrm{AA}$ & $61(27.0)$ & $108(29.8)$ & $0.87(0.60-1.26)$ & $0.89(0.61-1.29)$ & \\
\hline & rs1137101 & $\mathrm{GG}$ & $167(74.2)$ & 272 (75.6) & 1.00 (reference) & 1.00 (reference) & 0.649 \\
\hline & & $\mathrm{GA}$ & $56(24.9)$ & $81(22.5)$ & $1.13(0.76-1.67)$ & $1.13(0.76-1.67)$ & \\
\hline & & $\mathrm{AA}$ & $2(0.9)$ & 7 (1.9) & $0.47(0.10-2.27)$ & $0.49(0.10-2.41)$ & \\
\hline & & $G A+A A$ & $58(25.8)$ & $88(24.4)$ & $1.07(0.73-1.58)$ & $1.08(0.73-1.59)$ & \\
\hline & rs1938489 & $\mathrm{AA}$ & $198(88.0)$ & 325 (89.8) & 1.00 (reference) & 1.00 (reference) & 0.528 \\
\hline & & $A G$ & 26 (11.6) & $37(10.2)$ & $1.15(0.68-1.96)$ & $1.16(0.68-1.98)$ & \\
\hline & & GG & $1(0.4)$ & $0(0.0)$ & - & - & \\
\hline & & $A G+G G$ & $27(12.0)$ & $37(10.2)$ & $1.20(0.71-2.03)$ & $1.20(0.71-2.05)$ & \\
\hline & rs6673591 & $\mathrm{GG}$ & $172(76.4)$ & 278 (76.8) & 1.00 (reference) & 1.00 (reference) & 0.482 \\
\hline & & GA & $51(22.7)$ & $79(21.8)$ & $1.04(0.70-1.56)$ & $1.04(0.70-1.56)$ & \\
\hline & & $\mathrm{AA}$ & $2(0.9)$ & $5(1.4)$ & $0.65(0.12-3.37)$ & $0.71(0.14-3.73)$ & \\
\hline & & $G A+A A$ & 53 (23.6) & $84(23.2)$ & $1.02(0.69-1.51)$ & 1.02 (0.69-1.52) & \\
\hline & rs1137100 & $\mathrm{GG}$ & $142(62.8)$ & $234(64.8)$ & 1.00 (reference) & 1.00 (reference) & 0.742 \\
\hline & & $\mathrm{GA}$ & 73 (32.3) & $113(31.3)$ & 1.07 (0.74-1.53) & 1.07 (0.75-1.54) & \\
\hline & & $\mathrm{AA}$ & $11(4.9)$ & $14(3.9)$ & $1.30(0.57-2.93)$ & $1.33(0.58-3.02)$ & \\
\hline & & $\mathrm{GA}+\mathrm{AA}$ & $84(37.2)$ & $127(35.2)$ & $1.09(0.77-1.54)$ & $1.10(0.78-1.56)$ & \\
\hline & rs13306523 & $\mathrm{CC}$ & $209(92.1)$ & 339 (93.4) & 1.00 (reference) & 1.00 (reference) & 0.370 \\
\hline & & CT & $18(7.9)$ & $24(6.6)$ & $1.22(0.65-2.30)$ & $1.22(0.64-2.32)$ & \\
\hline & & $\Pi$ & $0(0.0)$ & $0(0.0)$ & - & - & \\
\hline & & $\mathrm{CT}+\mathrm{TT}$ & $18(7.9)$ & $24(6.6)$ & $1.22(0.65-2.30)$ & 1.22 (0.64-2.32) & \\
\hline \multirow[t]{8}{*}{ PPARG } & rs796313 & $G G$ & $80(35.6)$ & $121(33.2)$ & 1.00 (reference) & 1.00 (reference) & 0.275 \\
\hline & & GT & $114(50.7)$ & $183(50.3)$ & $0.94(0.65-1.36)$ & $0.91(0.62-1.31)$ & \\
\hline & & $\pi$ & $31(13.7)$ & $60(16.5)$ & $0.78(0.47-1.31)$ & $0.78(0.46-1.31)$ & \\
\hline & & $\mathrm{GT}+\pi$ & $145(64.4)$ & $243(66.8)$ & $0.90(0.64-1.28)$ & $0.87(0.61-1.24)$ & \\
\hline & rs17793951 & $\mathrm{AA}$ & $190(84.4)$ & $340(93.7)$ & 1.00 (reference) & 1.00 (reference) & 0.678 \\
\hline & & $A G$ & 35 (15.6) & $22(6.1)$ & $2.85(1.62-4.99)^{\star}$ & $2.94(1.67-5.19)^{\star}$ & \\
\hline & & $\mathrm{GG}$ & $0(0.0)$ & $1(0.3)$ & - & - & \\
\hline & & $A G+G G$ & 35 (15.6) & $23(6.3)$ & $2.72(1.56-4.75)$ & $2.81(1.61-4.92)$ & \\
\hline
\end{tabular}

${ }^{*}$ The $\mathrm{p}$ value $(\mathrm{p}=0.001)$ of trend analysis was statistically significant after Bonferroni correction.

${ }^{a}$ The number may not equal to the total number due to missing data.

${ }^{b}$ Unadjusted any variables.

${ }^{c}$ Adjusted for age and gender.

$P_{\text {HWE }}$ was the value of Hardy-Weinberg equilibrium test.

\section{RESULTS}

\section{Characteristics of Subjects}

To investigate the association of SNPs in $L E P, L E P R$, and PPARG with humoral response to influenza vaccine in the Chinese Han population, 592 subjects were recruited in this study including 227 low-responders and 365 responders. The general characteristics were shown in Supplementary Table 4. There was no significant difference in gender and age between the two groups.

\section{Comparison of Genotypic Frequencies of LEP, LEPR, and PPARG Between Low-Responders and Responders}

A total of 11 SNPs in $L E P, L E P R$, and PPARG were genotyped, and genotype distribution of all SNPs in the 592 subjects conformed to Hardy-Weinberg equilibrium $(p>0.05)$. The information regarding the genotyped SNPs was described in detail in Supplementary Table 1, in terms of chromosome positions, consequences, alleles, and minor allele frequencies. 


\section{PPARG rs17793951}

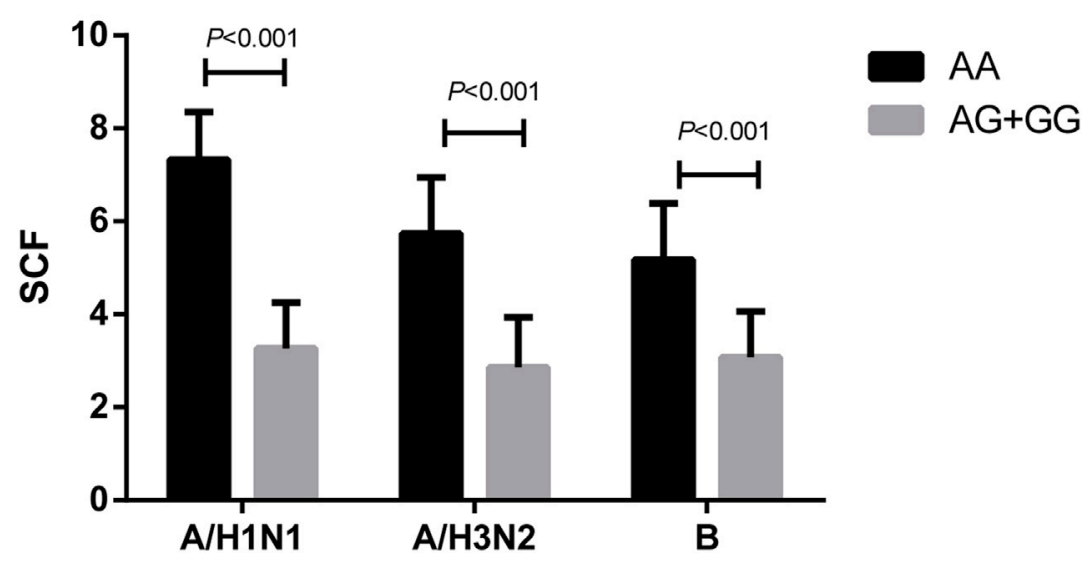

FIGURE 2 | Comparison the SCFs of different vaccine components between PPARG rs17793951 AA carriers and AG + GG carriers.

The MAFs of all SNPs were in accordance with those in the CHB population, which were reported by the 1000 Genome Project (phase 3). Furthermore, additive genetic model and dominant genetic model were performed to investigate the association between 11 tag SNPs and humoral response to influenza vaccine. Since few subjects were carrying PPARG rs17793951 GG genotype, we only compared the genotypic frequencies of $\mathrm{AA}$ and $\mathrm{AG}$ between the two groups in the additive genetic model. The results indicated the genotypic frequencies of PPARG rs17793951 AG in low-responders was higher than that in responders $(p=0.001)$, and the multivariate logistic regression analysis showed that AG genotype was correlated with a higher risk of low responsiveness to influenza vaccine adjusted for gender and age $(\mathrm{OR}=2.94$, 95\% CI $=1.67-5.19)$. This difference was still significant after the Bonferroni correction. In dominant genetic model analysis, the PPARG rs17793951 AG + GG genotype was related with lower response to influenza vaccine in both univariate logistic regression analysis $(\mathrm{OR}=2.71,95 \% \mathrm{CI}=1.56-4.75)$ and multivariate logistic regression analysis $(\mathrm{OR}=2.81,95 \% \mathrm{CI}=$ 1.61-4.92). However, no significant association of tag SNPs in $L E P$ and $L E P R$ with low responsiveness to influenza vaccine was found in our study (Table 1).

\section{PPARG rs17793951 AG + GG Carriers had Lower Antibody Fold Rises}

The comparison of SCFs of different vaccine components between PPARG rs17793951 AA carriers and AG + GG carriers was shown in Figure 2. The antibody fold rises of PPARG rs17793951 AG + GG genotype carriers were significantly lower than that of AA genotype carriers against all three components $(p<0.001)$. The specific SCF values were shown in Supplementary Table 5.

\section{Stratification Analysis of the Association Between Responsiveness to Influenza Vaccine and LEPR/PPARG Polymorphisms}

The stratified analysis found that LEPR rs6673591 GA + AA genotype was related to low responsiveness to influenza vaccine in males $(\mathrm{OR}=1.96,95 \% \mathrm{CI}=1.05-3.67)$. However, the contrary correlation was found in females even if without statistical significance $(\mathrm{OR}=0.63,95 \% \mathrm{CI}$ $=0.37-1.08)$. We also found that the genotypic frequencies of PPARG rs17793951 AG + GG in low-responders were higher than that in responders both in males and females, but it was significant only in females (Table 2).

\section{LD and Haplotype Analysis of SNPs in LEP, LEPR, and PPARG}

The linkage disequilibrium (LD) structures of two SNPs in $L E P$, seven SNPs in $L E P R$, and two SNPs in PPARG were shown in Supplementary Tables 6-8, and they were not in LD. Haplotypes in $L E P$ were not correlated with response to influenza vaccine (Supplementary Table 9). Furthermore, compared with haplotype CGGAGGC, we found that the haplotype CAAAAAC in the $L E P R$ was positively associated with responsiveness to influenza vaccine $(p=0.010, \mathrm{OR}=0.34$, 95\% CI $=0.15-0.77$, Table 3). Haplotype blocks were also built between PPARG rs796313 and rs17793951, and four haplotypes were identified (Table 4). Compared with haplotype GA, the uncommon haplotype TG was associated with low responsiveness to influenza vaccine $(p=0.002, \mathrm{OR}=$ $3.85,95 \% \mathrm{CI}=1.67-9.09)$. Moreover, no gene-gene interaction among 11 SNPs of $L E P, L E P R$, and PPARG in the GMDR method was found (Supplementary Table 10). 
TABLE 2 | Association of SNPs in LEPR and PPARG with influenza vaccine-induced humoral responses stratified by gender.

\begin{tabular}{|c|c|c|c|c|c|c|c|c|}
\hline \multirow[t]{2}{*}{ Gene } & \multirow[t]{2}{*}{ SNP ID } & \multirow[t]{2}{*}{ Genotype } & \multicolumn{3}{|c|}{ Female $(\mathbf{N}=359)$} & \multicolumn{3}{|c|}{ Male $(\mathbf{N}=233)$} \\
\hline & & & $\begin{array}{c}\text { Low-responders } \mathrm{N}^{\mathrm{a}} \\
(\%)\end{array}$ & $\begin{array}{c}\text { Responders } \mathrm{N}^{\mathrm{a}} \\
(\%)\end{array}$ & $\begin{array}{c}\text { OR } \\
(95 \% \mathrm{Cl})^{\mathrm{b}}\end{array}$ & $\begin{array}{l}\text { Low-responders } N^{*} \\
(\%)\end{array}$ & $\begin{array}{c}\text { Responders } N^{*} \\
(\%)\end{array}$ & $\begin{array}{c}\text { OR } \\
(95 \% \mathrm{Cl})^{\mathrm{b}}\end{array}$ \\
\hline \multirow[t]{2}{*}{ LEPR } & rs6673591 & $\mathrm{GG}$ & $110(81.5)$ & $166(74.4)$ & 1.00 (reference) & 62 (68.9) & $112(80.6)$ & 1.00 (reference) \\
\hline & & $\mathrm{GA}+\mathrm{AA}$ & $25(18.5)$ & 57 (25.6) & $0.63(0.37-1.08)$ & $28(31.1)$ & 27 (19.4) & $1.96(1.05-3.67)$ \\
\hline \multirow[t]{2}{*}{ PPARG } & rs17793951 & $\mathrm{AA}$ & $112(83.0)$ & 209 (93.7) & 1.00 (reference) & $78(86.7)$ & 131 (93.6) & 1.00 (reference) \\
\hline & & $A G+G G$ & $23(17.0)$ & $14(6.3)$ & $3.28(1.61-6.67)$ & 12 (13.3) & $9(6.4)$ & $2.18(0.87-5.46)$ \\
\hline
\end{tabular}

${ }^{a}$ The number may not equal to the total number due to missing data.

${ }^{b}$ Adjusted for age.

\begin{tabular}{|c|c|c|c|c|c|c|c|c|c|c|c|c|}
\hline \multirow{2}{*}{$\begin{array}{l}\text { LEPR } \\
\text { Haplotype }\end{array}$} & \multicolumn{7}{|c|}{ SNPa } & \multicolumn{3}{|c|}{ Frequency } & \multirow{2}{*}{$\begin{array}{c}\text { OR } \\
(95 \% \mathrm{Cl})\end{array}$} & \multirow[t]{2}{*}{$P$} \\
\hline & 1 & 2 & 3 & 4 & 5 & 6 & 7 & Total & Low-responders & Responders & & \\
\hline 1 & $\mathrm{C}$ & $G$ & $G$ & $A$ & $G$ & $G$ & $\mathrm{C}$ & 0.63 & 0.65 & 0.63 & 1.00 (reference) & - \\
\hline 2 & $G$ & $G$ & $G$ & $A$ & $G$ & $G$ & C & 0.06 & 0.05 & 0.06 & $0.95(0.56-1.64)$ & 0.860 \\
\hline 3 & C & $A$ & $A$ & $A$ & $A$ & $A$ & $\mathrm{C}$ & 0.04 & 0.02 & 0.06 & $0.34(0.15-0.77)$ & 0.010 \\
\hline 4 & $\mathrm{C}$ & $G$ & $A$ & $A$ & $A$ & $A$ & $\mathrm{C}$ & 0.04 & 0.05 & 0.03 & $1.54(0.76-3.03)$ & 0.230 \\
\hline 5 & $\mathrm{G}$ & $A$ & $G$ & $A$ & $G$ & $G$ & $\mathrm{C}$ & 0.03 & 0.03 & 0.04 & $0.74(0.35-1.59)$ & 0.440 \\
\hline 6 & C & $G$ & $G$ & $\mathrm{G}$ & G & A & $\mathrm{C}$ & 0.03 & 0.02 & 0.03 & $0.66(0.27-1.64)$ & 0.370 \\
\hline 7 & $\mathrm{C}$ & $A$ & $G$ & $A$ & $G$ & $G$ & $\mathrm{C}$ & 0.03 & 0.02 & 0.03 & $0.85(0.36-2.00)$ & 0.720 \\
\hline 8 & $\mathrm{C}$ & $G$ & $G$ & $A$ & $G$ & $G$ & $\mathrm{~T}$ & 0.02 & 0.02 & 0.02 & $1.49(0.60-3.70)$ & 0.390 \\
\hline 9 & $G$ & $G$ & $A$ & $A$ & $A$ & $A$ & $\mathrm{C}$ & 0.02 & 0.03 & 0.01 & $2.13(0.76-5.88)$ & 0.150 \\
\hline 10 & C & $A$ & $G$ & $A$ & $G$ & $A$ & C & 0.02 & 0.02 & 0.01 & $1.79(0.68-4.76)$ & 0.240 \\
\hline 11 & $G$ & $A$ & $A$ & $A$ & $A$ & $A$ & C & 0.01 & 0.02 & 0.01 & $3.85(0.68-20.00)$ & 0.130 \\
\hline 12 & $\mathrm{C}$ & $A$ & $G$ & $A$ & $G$ & $G$ & $\mathrm{~T}$ & 0.01 & 0.01 & 0.01 & $1.12(0.34-3.85)$ & 0.850 \\
\hline 13 & $\mathrm{C}$ & $G$ & $A$ & $A$ & $G$ & $G$ & C & 0.01 & 0.01 & 0.01 & $0.93(0.25-3.45)$ & 0.910 \\
\hline 14 & $G$ & $G$ & $G$ & $G$ & $G$ & $A$ & C & 0.01 & 0.01 & 0.01 & $0.60(0.17-2.08)$ & 0.420 \\
\hline Rare $^{b}$ & - & - & - & - & - & - & - & - & - & - & - & - \\
\hline
\end{tabular}

SNPa: 1-rs1327118, 2-rs7602, 3-rs1137101, 4-rs1938489, 5-rs6673591, 6-rs1137100, and 7-rs13306523.

Rare $^{b}$ : Haplotypes with frequencies $<0.01$.

TABLE 4 | Association between haplotypes of PPARG and low responsiveness to influenza vaccine.

\begin{tabular}{|c|c|c|c|c|c|c|c|}
\hline \multirow[t]{2}{*}{ PPARG Haplotype } & \multicolumn{3}{|c|}{ SNP } & \multicolumn{2}{|c|}{ Frequency } & \multirow[t]{2}{*}{ OR $(95 \% \mathrm{Cl})$} & \multirow[t]{2}{*}{$\boldsymbol{P}$} \\
\hline & rs796313 & rs17793951 & Total & Low-responders & Responders & & \\
\hline 1 & $G$ & $A$ & 0.57 & 0.59 & 0.56 & 1.00 (reference) & - \\
\hline 2 & $\mathrm{~T}$ & $A$ & 0.38 & 0.33 & 0.40 & $0.78(0.60-1.02)$ & 0.073 \\
\hline 3 & $\mathrm{~T}$ & $G$ & 0.03 & 0.06 & 0.01 & 3.85 (1.67-9.09) & 0.002 \\
\hline 4 & $G$ & $G$ & 0.02 & 0.02 & 0.02 & $1.08(0.36-3.23)$ & 0.900 \\
\hline
\end{tabular}

\section{DISCUSSION}

To investigate the roles of $L E P, L E P R$, and PPARG in the humoral immune response to influenza vaccine, we conducted an association study to compare the genotypic frequencies of 11 tag SNPs in these genes between the two groups in the Chinese Han population. The most significant result was that PPARG rs17793951 AG + GG genotype was associated with low responsiveness to influenza vaccine, and we also observed that LEPR rs6673591 GA + AA genotype was correlated with low responsiveness to influenza vaccine only in males by stratified analysis.
Leptin is a protein hormone $(16 \mathrm{kDa})$ secreted by adipocytes. It directly acts on $\mathrm{CD} 4^{+} \mathrm{T}$ helper cells, $\mathrm{CD} 8^{+}$cytotoxic $\mathrm{T}$ cells, and $\mathrm{B}$ cells by interacting with the membrane-bound leptin receptor (Papathanassoglou et al., 2006). A recent study reported that increased concentrations of leptin may help maintain the naive $\mathrm{T}$ cell pool in the elderly (Chen et al., 2010). Evidence also showed that leptin signaling regulates $\mathrm{B}$ cell homeostasis through activating Bcl-2 and cyclin D1 (Lam et al., 2010). Although previous studies suggested that leptin played important roles in regulation specific-IgG antibody levels of influenza vaccine and clearance of influenza virus (Zhang et al., 2013; Frasca et al., 2020), we didn't find significant associations between tag SNPs in 
$L E P$ and $L E P R$ and antibody response to influenza vaccine, which was in agreement with a previous report (Ovsyannikova et al., 2014). In general, there were few studies focused on the relationship between leptin and influenza vaccines, and the underlying mechanisms were still unclear.

As we know, PPARG is a lipid-activated nuclear receptor expressed on the surface of macrophages, dendritic cells, and regulatory T cells (Odegaard et al., 2007; Tuna et al., 2014; Khare et al., 2015). A recent study has demonstrated that three SNPs in PPARG were significantly related to the baseline level of immunomodulator, Vitamin D, and the authors presumed that PPARG and Vitamin D receptors may interact with each other and participate in immune response to influenza vaccine (Sadarangani et al., 2016). Furthermore, PPARG help to restrict pulmonary inflammation and promote recovery from influenza virus infection (Huang et al., 2019). These studies suggested that PPARG may play a role in immune response to influenza virus or vaccine. Indeed, our study found that $P P A R G$ rs17793951 was associated with immune response to influenza vaccine in additive genetic model and dominant genetic model. The risk of low responsiveness to influenza vaccine in $P P A R G$ rs17793951 AG + GG genotype was almost 3 times higher than that of AA genotype in multivariate logistic regression analysis. Subjects carrying AG + GG genotype had a lower fold-rise of antibody titer to all vaccine components. Similarly, an American study (Ovsyannikova et al., 2014) used the linear correlation methods to explore the association of leptin-related genes variants with the antibody response to influenza vaccine in 50-80-year-old Caucasians, and they found that PPARG rs17793951G minor allele was associated with the A/H1N1 HAI antibody titers. Although there were some different aspects on subjects' populations and study design between this research and our study, the results both showed that PPARG rs17793951 variants might play an important role in immune response to influenza vaccine. Unfortunately, there was still no research to demonstrate the exact mechanism of this association. What's more, the rs 17793951 was located in intron two of $P P A R G$ and could bind to CCCTC-binding factor (CTCF) in the functional annotations of HaploReg V.4.1. Specifically, CTCF is a transcription factor and has many effects on chromosome segregation and regulation of genes expression as an insulator (Kang and Lee, 2021; Pongubala and Murre, 2021). Therefore, we hypothesized that the rs17793951 A to $G$ change may suppress $P P A R G$ transcription by enhancing the binding ability of this site to CTCF, resulting in a downregulation of immune response consequently. More research is needed to verify the association of PPARG rs17793951 with humoral response to influenza vaccine and reveal its physiological mechanisms.

Gender is an important factor affecting immune response to influenza vaccine. Stratified analysis implicated that LEPR rs6673591 GA + AA genotype had an approximately doubled risk of low responsiveness compared with AA genotype in males. On the contrary, LEPR rs6673591 GA + AA genotype in females tends to correlate with higher antibody response, but the difference was not statistically significant. Moreover, $P P A R G$ rs17793951 AG + GG genotype was associated with a higher risk of low responsiveness to influenza vaccine in males and females, but this effect was significant only in females. An article also reported gender difference in the association of genetic polymorphisms with immune response to Japanese encephalitis vaccine (Yao et al., 2020). We speculated that some immune-suppressive behaviors more commonly in men may mask the correlation of genotypes with response to influenza vaccine, such as smoking (Godoy et al., 2018). Additionally, women have higher concentrations of leptin and estrogen normally (Thomas et al., 2000), with up-regulation of immune response, thereby there were modification effects of gender on the association of SNPs with humoral response to influenza vaccine. It is worthy to explore the mechanisms of gender difference on the association of SNPs with antibody response to influenza vaccine.

Our study demonstrated that the haplotype CAAAAAC in the LEPR gene composed of seven tag SNPs was positively correlated with vaccine response compared with the CGGAGGC haplotype, which was a joint contribution of rs7602 in $3^{\prime} \mathrm{UTR}$, rs6673591 in intron, rs1137101 and rs1137100 in exons, although no significant association was found in single SNP analysis. This discordance may be explained by the fact that vaccine response is a complex process involving multiple factors and each of the SNPs has a tiny influence on immune response. Moreover, when haplotypes were constructed with PPARG rs17793951 and rs796313 SNPs, we found a more significant association between TG haplotype with poor responsiveness to influenza vaccine, which was consistent with single SNP analysis. The results further suggested that PPARG rs17793951 polymorphism played an important role in immune response to influenza vaccine. Additional studies are required to verify our hypotheses in different populations.

As far as we know, this is the first research to examine the association between SNPs in $L E P, L E P R$, and PPARG and the humoral response of $\mathrm{A} / \mathrm{H} 1 \mathrm{~N} 1, \mathrm{~A} / \mathrm{H} 3 \mathrm{~N} 2$, and $\mathrm{B}$ vaccine components in the Chinese Han population. There are still several limitations in our study. A relatively small sample of subjects was used in our study. However, our subjects were carefully selected based on seroconversion to all or none of vaccine components with sufficient statistical power, and the MAFs of tag SNPs were in accordance with that of the 1000 Genome Project (phase 3). Although we adjusted for the known influencing factors to evaluate this association, such as age and gender, there may exist other uncollected factors affecting immune response to influenza vaccine. In addition, our study only assessed the antibody level on the 28th day post-vaccination when it reached a peak, yet antibody level will change over time. Therefore, future studies are required to investigate this association through longer-term observation and provide more sufficiently theoretical evidence for the development of universal influenza vaccines.

\section{CONCLUSION}

The present study demonstrated the association between PPARG rs17793951 AG + GG genotype and low responsiveness to influenza vaccine in the Chinese Han population. 


\section{DATA AVAILABILITY STATEMENT}

The original contributions presented in the study are included in the article/Supplementary Material, further inquiries can be directed to the corresponding authors.

\section{ETHICS STATEMENT}

The studies involving human participants were reviewed and approved by the Ethics Review Committee of the National Institute for Viral Disease Control and Prevention (NIVDC, assurance number, 200916). Written informed consent to participate in this study was provided by the participants' legal guardian/next of kin.

\section{AUTHOR CONTRIBUTIONS}

DW and YS conceived and designed the research. HW and YC collected the blood samples and epidemiology data. ML, SZ, SW, and YC performed the experiments. ML and SZ analyzed the data. ML wrote the article. DW, YS, SW, and SZ commented on the article. All authors not only read and approved the final version of the article, but also agreed to be accountable for the content of the work.

\section{REFERENCES}

Agrawal, S., Gollapudi, S., Su, H., and Gupta, S. (2011). Leptin Activates Human B Cells to Secrete TNF- $\alpha$, IL-6, and IL-10 via JAK2/STAT3 and p38MAPK/ ERK1/2 Signaling Pathway. J. Clin. Immunol. 31 (3), 472-478. doi:10.1007/ s10875-010-9507-1

Chen, J., Li, J., Lim, F. C., Wu, Q., Douek, D. C., Scott, D. K., et al. (2010). Maintenance of Naïve CD8 T Cells in Nonagenarians by Leptin, IGFBP3 and T3. Mech. Ageing Dev. 131 (1), 29-37. doi:10.1016/j.mad.2009.11.003

Dhakal, S., and Klein, S. L. (2019). Host Factors Impact Vaccine Efficacy: Implications for Seasonal and Universal Influenza Vaccine Programs. J. Virol. 93 (21), e00797-00719. doi:10.1128/jvi.00797-19

Francisco, V., Pino, J., Campos-Cabaleiro, V., Ruiz-Fernández, C., Mera, A., Gonzalez-Gay, M. A., et al. (2018). Obesity, Fat Mass and Immune System: Role for Leptin. Front. Physiol. 9, 640. doi:10.3389/fphys.2018.00640

Franco, L. M., Bucasas, K. L., Wells, J. M., Niño, D., Wang, X., Zapata, G. E., et al. (2013). Integrative Genomic Analysis of the Human Immune Response to Influenza Vaccination. Elife 2, e00299. doi:10.7554/eLife.00299

Frasca, D., Diaz, A., Romero, M., and Blomberg, B. B. (2020). Leptin Induces Immunosenescence in Human B Cells. Cell Immunol. 348, 103994. doi:10.1016/ j.cellimm.2019.103994

Godoy, P., Castilla, J., Soldevila, N., Mayoral, J. M., Toledo, D., Martín, V., et al. (2018). Smoking May Increase the Risk of Influenza Hospitalization and Reduce Influenza Vaccine Effectiveness in the Elderly. Eur. J. Public Health 28 (1), 150-155. doi:10.1093/eurpub/ckx130

Huang, S., Jiang, L., Cheon, I. S., and Sun, J. (2019). Targeting Peroxisome Proliferator-Activated Receptor-Gamma Decreases Host Mortality after Influenza Infection in Obese Mice. Viral Immunol. 32 (4), 161-169. doi:10.1089/vim.2019.0016

Iuliano, A. D., Roguski, K. M., Chang, H. H., Muscatello, D. J., Palekar, R., Tempia, S., et al. (2018). Estimates of Global Seasonal Influenza-Associated Respiratory Mortality: a Modelling Study. Lancet 391 (10127), 1285-1300. doi:10.1016/ s0140-6736(17)33293-2

Joffe, Y. T., and Houghton, C. A. (2016). A Novel Approach to the Nutrigenetics and Nutrigenomics of Obesity and Weight Management. Curr. Oncol. Rep. 18 (7), 43. doi:10.1007/s11912-016-0529-6

\section{FUNDING}

This work was supported by the Shenzhen science and technology program (Grant number: kqtd20180411143323605) and The National Natural Science Foundation of China (Grant number: 82041043).

\section{ACKNOWLEDGMENTS}

We would like to thank the staff of the Center for Disease Control and Prevention of Xinjiang Uygur Autonomous Region and Yunnan Province for their help in recruiting subjects and collecting samples. We also appreciate all of the subjects' participation and cooperation in this project.

\section{SUPPLEMENTARY MATERIAL}

The Supplementary Material for this article can be found online at: https://www.frontiersin.org/articles/10.3389/fgene.2021.725538/ full\#supplementary-material

Kang, M. A., and Lee, J.-S. (2021). A Newly Assigned Role of CTCF in Cellular Response to Broken DNAs. Biomolecules 11 (3), 363. doi:10.3390/ biom 11030363

Khare, A., Chakraborty, K., Raundhal, M., Ray, P., and Ray, A. (2015). Cutting Edge: Dual Function of PPAR $\gamma$ in CD11c+ Cells Ensures Immune Tolerance in the Airways. J.I. 195 (2), 431-435. doi:10.4049/jimmunol.1500474

Kim, S. Y., Lim, J. H., Choi, S. W., Kim, M., Kim, S.-T., Kim, M.-S., et al. (2010). Preferential Effects of Leptin on CD4 T Cells in central and Peripheral Immune System Are Critically Linked to the Expression of Leptin Receptor. Biochem. Biophysical Res. Commun. 394 (3), 562-568. doi:10.1016/j.bbrc.2010.03.019

Lam, Q. L. K., Wang, S., Ko, O. K. H., Kincade, P. W., and Lu, L. (2010). Leptin Signaling Maintains B-Cell Homeostasis via Induction of Bcl-2 and Cyclin D1. Proc. Natl. Acad. Sci. 107 (31), 13812-13817. doi:10.1073/pnas.1004185107

Narang, V., Lu, Y., Tan, C., Camous, X. F. N., Nyunt, S. Z., Carre, C., et al. (2018). Influenza Vaccine-Induced Antibody Responses Are Not Impaired by Frailty in the Community-Dwelling Elderly with Natural Influenza Exposure. Front. Immunol. 9, 2465. doi:10.3389/fimmu.2018.02465

Odegaard, J. I., Ricardo-Gonzalez, R. R., Goforth, M. H., Morel, C. R., Subramanian, V., Mukundan, L., et al. (2007). Macrophage-specific PPAR $\gamma$ Controls Alternative Activation and Improves Insulin Resistance. Nature 447 (7148), 1116-1120. doi:10.1038/nature05894

Ovsyannikova, I. G., White, S. J., Larrabee, B. R., Grill, D. E., Jacobson, R. M., and Poland, G. A. (2014). Leptin and Leptin-Related Gene Polymorphisms, Obesity, and Influenza A/H1N1 Vaccine-Induced Immune Responses in Older Individuals. Vaccine 32 (7), 881-887. doi:10.1016/j.vaccine.2013.12.009

Papathanassoglou, E., El-Haschimi, K., Li, X. C., Matarese, G., Strom, T., and Mantzoros, C. (2006). Leptin Receptor Expression and Signaling in Lymphocytes: Kinetics during Lymphocyte Activation, Role in Lymphocyte Survival, and Response to High Fat Diet in Mice. J. Immunol. 176 (12), 7745-7752. doi:10.4049/jimmunol.176.12.7745

Poland, G. A., Ovsyannikova, I. G., and Jacobson, R. M. (2008). Immunogenetics of Seasonal Influenza Vaccine Response. Vaccine 26 (Suppl. 4Suppl 4), D35-D40. doi:10.1016/j.vaccine.2008.07.065

Pongubala, J. M. R., and Murre, C. (2021). Spatial Organization of Chromatin: Transcriptional Control of Adaptive Immune Cell Development. Front. Immunol. 12, 633825. doi:10.3389/fimmu.2021.633825 
Sadarangani, S. P., Ovsyannikova, I. G., Goergen, K., Grill, D. E., and Poland, G. A. (2016). Vitamin D, Leptin and Impact on Immune Response to Seasonal Influenza A/H1N1 Vaccine in Older Persons. Hum. Vaccin. Immunother. 12 (3), 691-698. doi:10.1080/21645515.2015.1097015

Soedjatmiko, S., Medise, B. E., Gunardi, H., Sekartini, R., Satari, H. I., Hadinegoro, S. R., et al. (2018). Immunogenicity and Safety of a Trivalent Influenza HA Vaccine in Indonesian Infants and Children. Vaccine 36 (16), 2126-2132. doi:10.1016/j.vaccine.2018.02.114

Thomas, T., Burguera, B., Melton, L. J., 3rd, Atkinson, E. J., O’Fallon, W. M., Riggs, B. L., et al. (2000). Relationship of Serum Leptin Levels with Body Composition and Sex Steroid and Insulin Levels in Men and Women. Metabolism 49 (10), 1278-1284. doi:10.1053/meta.2000.9519

Tuna, H., Avdiushko, R. G., Sindhava, V. J., Wedlund, L., Kaetzel, C. S., Kaplan, A. M., et al. (2014). Regulation of the Mucosal Phenotype in Dendritic Cells by PPAR $\gamma$ : Role of Tissue Microenvironment. J. Leukoc. Biol. 95 (3), 471-485. doi:10.1189/jlb.0713408

World Health Organization (2011). Manual for the Laboratory Diagnosis and Virological Surveillance of Influenza. Geneva, Switzerland: World Health Organization. Available at: https://apps.who.int/iris/handle/10665/44518 (Accessed June 12, 2021).

Yao, Y., Xu, X., Li, Y., Wang, X., Yang, H., Chen, J., et al. (2020). Study of the Association of Seventeen Single Nucleotide Polymorphisms and Their Haplotypes in the TNF- $\alpha$, IL-2, IL-4 and IL-10 Genes with the Antibody Response to Inactivated Japanese Encephalitis Vaccine. Hum. Vaccin. Immunother. 16 (10), 2449-2455. doi:10.1080/ 21645515.2020 .1724743
Zhang, A. J. X., To, K. K. W., Li, C., Lau, C. C. Y., Poon, V. K. M., Chan, C. C. S., et al. (2013). Leptin Mediates the Pathogenesis of Severe 2009 Pandemic Influenza $\mathrm{A}(\mathrm{H} 1 \mathrm{~N} 1)$ Infection Associated with Cytokine Dysregulation in Mice with Diet-Induced Obesity. J. Infect. Dis. 207 (8), 1270-1280. doi:10.1093/infdis/jit031

Conflict of Interest: The authors declare that the research was conducted in the absence of any commercial or financial relationships that could be construed as a potential conflict of interest.

Publisher's Note: All claims expressed in this article are solely those of the authors and do not necessarily represent those of their affiliated organizations, or those of the publisher, the editors and the reviewers. Any product that may be evaluated in this article, or claim that may be made by its manufacturer, is not guaranteed or endorsed by the publisher.

Copyright (c) $2021 \mathrm{Li}$, Wei, Zhong, Cheng, Wen, Wang and Shu. This is an openaccess article distributed under the terms of the Creative Commons Attribution License (CC BY). The use, distribution or reproduction in other forums is permitted, provided the original author(s) and the copyright owner(s) are credited and that the original publication in this journal is cited, in accordance with accepted academic practice. No use, distribution or reproduction is permitted which does not comply with these terms. 